\title{
Predictive Value of FOLFOX-Based Regimen, Long Interval, Hemoglobin Levels and Clinical Negative Nodal Status, and Postchemoradiotherapy CEA Levels for Pathological Complete Response in Patients with Locally Advanced Rectal Cancer after Neoadjuvant Chemoradiotherapy
}

\author{
Chun-Ming Huang, ${ }^{1,2,3,4}$ Ching-Wen Huang, ${ }^{3,5,6}$ Cheng-Jen Ma, ${ }^{5}$ Yung-Sung Yeh, ${ }^{5,7,8}$ \\ Wei-Chih Su, ${ }^{5}$ Tsung-Kun Chang, ${ }^{5}$ Hsiang-Lin Tsai, ${ }^{5,6}$ Suh-Hang Juo, ${ }^{7}$ \\ Ming-Yii Huang $\mathbb{D}^{2,4}$ and Jaw-Yuan Wang $\mathbb{D}^{3,5,6,7,9}$ \\ ${ }^{1}$ Department of Radiation Oncology, Kaohsiung Municipal Ta-Tung Hospital, Kaohsiung Medical University, Kaohsiung, Taiwan \\ ${ }^{2}$ Department of Radiation Oncology, Kaohsiung Medical University Hospital, Kaohsiung Medical University, Kaohsiung, Taiwan \\ ${ }^{3}$ Graduate Institute of Medicine, College of Medicine, Kaohsiung Medical University, Kaohsiung, Taiwan \\ ${ }^{4}$ Department of Radiation Oncology, Faculty of Medicine, College of Medicine, Kaohsiung Medical University, \\ Kaohsiung, Taiwan \\ ${ }^{5}$ Division of Colorectal Surgery, Department of Surgery, Kaohsiung Medical University Hospital, Kaohsiung Medical University, \\ Kaohsiung, Taiwan \\ ${ }^{6}$ Department of Surgery, Faculty of Medicine, College of Medicine, Kaohsiung Medical University, Kaohsiung, Taiwan \\ ${ }^{7}$ Graduate Institute of Clinical Medicine, College of Medicine, Kaohsiung Medical University, Kaohsiung, Taiwan \\ ${ }^{8}$ Division of Trauma and Critical Care, Department of Surgery, Kaohsiung Medical University Hospital, \\ Kaohsiung Medical University, Kaohsiung, Taiwan \\ ${ }^{9}$ Center for Cancer Research, Kaohsiung Medical University, Kaohsiung, Taiwan
}

Correspondence should be addressed to Ming-Yii Huang; miyihu@gmail.com and Jaw-Yuan Wang; cy614112@ms14.hinet.net

Received 5 July 2019; Revised 12 September 2019; Accepted 5 November 2019; Published 28 January 2020

Academic Editor: Francesca De Felice

Copyright (c) 2020 Chun-Ming Huang et al. This is an open access article distributed under the Creative Commons Attribution License, which permits unrestricted use, distribution, and reproduction in any medium, provided the original work is properly cited.

We aimed to identify predictors of a pathological complete response (pCR) in patients with locally advanced rectal cancer (LARC) following a multimodality therapy. We retrospectively reviewed 236 patients with LARC treated with neoadjuvant chemoradiotherapy (CRT) followed by radical resection from January 2011 to December 2017. Patients were administered CRT, which comprised radiotherapy and chemotherapy with an oxaliplatin plus 5-fluorouracil- or fluoropyrimidine-based regimen. Clinical factors were correlated with treatment response. The multivariate logistic regression revealed that a negative nodal stage (odds ratio $(\mathrm{OR})=3.2, P=0.0135)$, a high hemoglobin level $(>10 \mathrm{~g} / \mathrm{dL})$ during neoadjuvant $\mathrm{CRT}(\mathrm{OR}=3.067, P=0.0125)$, an oxaliplatin-containing neoadjuvant CRT $(\mathrm{OR}=5.385, P=0.0044)$, a long interval ( $>8$ weeks) between radiotherapy and surgery $(\mathrm{OR}=1.135, P=0.0469)$, and a post-CRT CEA $\leq 2 \mathrm{ng} / \mathrm{mL}(\mathrm{OR}=2.891, P=0.0233)$ were the independent predictors of increased pCR rates. The prediction nomogram was developed according to the above independent variables. The concordance index was 0.74 , and the calibration curve showed good agreement. In summary, negative nodal stages, high hemoglobin levels during treatment, oxaliplatin-containing neoadjuvant therapy, a long radiotherapy-surgery interval ( $>8$ weeks), and post-CRT CEA levels $\leq 2 \mathrm{ng} / \mathrm{mL}$ were favorable predictors of a pCR. This prediction nomogram might be crucial for patients with LARC undergoing a multimodality therapy. 


\section{Introduction}

For locally advanced rectal cancer (LARC), neoadjuvant chemoradiotherapy (CRT) has been the standard treatment because it provides high local control, low treatment toxicity, high rates of sphincter preservation, and improved diseasefree survival (DFS) [1-3]. However, the literature shows inconsistent treatment response to neoadjuvant CRT, ranging from a pathological complete response (pCR) to total resistance. A pCR to neoadjuvant CRT has been associated with a low rate of recurrence and favorable survival, but the incidence of pCR has ranged from $10 \%$ to $30 \%$ [2-8]. Therefore, how to identify patients who can benefit the most from neoadjuvant CRT remains unresolved.

Although numerous methods to predict a pCR before surgery have been investigated, the predictors identified are not consistent across studies $[9,10]$. Some clinical parameters and molecular biomarkers have been reported to be predictors of a pCR to neoadjuvant CRT for patients with LARC [9-11]. Several clinical factors, such as pretreatment T or $\mathrm{N}$ stage, serum carcinoembryonic antigen (CEA), chemotherapy regimen, and radiation dose, have been associated with a pCR $[5,12-16]$. Few studies have investigated the effect of treatment-related hematologic toxicity on a pCR. Therefore, we aimed to identify a correlation between hematologic toxicity with $\mathrm{pCR}$ in this study.

Identifying the predictive factors of $\mathrm{pCR}$ is helpful because of the efficacy and safety of watch-and-wait approaches for patients who achieved a $\mathrm{PCR}$ to neoadjuvant CRT [17]. The benefit of watch-and-wait approaches is the avoidance of a morbid radical resection without compromising tumor control [18]. The dilemma we encountered is how to identify the patients who might be benefited by watch-and-wait approaches. The aim of this study was to identify the predictive factors of $\mathrm{pCR}$ in patients with LARC who may benefit from watch-and-wait strategies.

\section{Materials and Methods}

2.1. Patients. We analyzed 248 patients with histopathologically proven locally advanced rectal adenocarcinoma (T3-4 or $\mathrm{N}+$ ) who received neoadjuvant CRT followed by total mesorectal excision (TME) in a single institute from January 2011 to December 2017. The exclusion criteria involved previous or synchronous malignancies other than nonmelanoma skin cancer, local excision of rectal tumor, and a history of pelvic irradiation. Twelve patients were excluded from this analysis because of incomplete neoadjuvant CRT $(n=4)$, refusal of surgery $(n=3)$, unresectable tumors at surgery $(n=3)$, and local excision of primary tumor only $(n=2)$. The remaining 236 LARC patients without evidence of distant metastasis were enrolled. Our institutional review board approved this study. Pretreatment evaluation included a medical history review, physical examination, colonoscopy with tumor biopsy, chest radiography, abdominal computed tomography $(\mathrm{CT})$, pelvic magnetic resonance imaging (MRI), a serum CEA assessment, and routine laboratory studies.
2.2. Chemotherapy. A fluoropyrimidine-based regimen was delivered to 95 patients. The regimen included (1) 5-fluorouracil (5-FU; $350 \mathrm{mg} / \mathrm{m}^{2}$, intravenous bolus) and leucovorin $\left(20 \mathrm{mg} / \mathrm{m}^{2}\right.$, intravenous bolus) on days 1 to 5 and days 21 to 25 of irradiation, once every 2 weeks or (2) 6 cycles of capecitabine $850 \mathrm{mg} / \mathrm{m}^{2}$ twice daily for 14 days, followed by 7 days of rest after each cycle [19]. For the rest of the 141 patients, a biweekly schedule of FOLFOX was prescribed. Each cycle of FOLFOX consisted of oxaliplatin $\left(85 \mathrm{mg} / \mathrm{m}^{2}\right)$ on day 1 , folinic acid $\left(400 \mathrm{mg} / \mathrm{m}^{2}\right)$, and a $46-\mathrm{h}$ infusion of 5FU $\left(2800 \mathrm{mg} / \mathrm{m}^{2}\right)$ repeated every 2 weeks. After irradiation completion, all patients continued biweekly FOLFOX until 2 to 3 weeks before surgery.

2.3. Radiotherapy. Patients were simulated with CT in a supine position with a customized thermoplastic immobilization device. All patients were instructed to void their bladder and then drink $300 \mathrm{ml}$ of water $30 \mathrm{~min}$ before simulation and irradiation. The total radiation dose was delivered in a range of 45 to $50.4 \mathrm{~Gy}$ using a daily fraction of 1.8 to $2.0 \mathrm{~Gy}$. We added a 1.5 to $2 \mathrm{~cm}$ clinical target margin to cover the gross tumor volume. Beyond the clinical target margin, we added a planning target margin of 1 to $1.5 \mathrm{~cm}$. All patients received external-beam radiotherapy with either 3dimensional conformal or intensity-modulated radiation therapy.

2.4. Surgery and Pathology Review. All patients underwent TME after neoadjuvant CRT completion [19]. There were 207 patients (87.7\%) undergoing low anterior resection with colorectal or coloanal anastomosis and 29 patients (12.3\%) receiving abdominoperineal resection. Two experienced pathologists, who are specialized in colorectal cancer, assessed the tumor response to neoadjuvant CRT. A pCR was defined as the absence of any viable cancer cells in the primary tumor and nodes (ypT0N0) in resected specimens after neoadjuvant treatment. More than $10 \%$ of mucins in resection specimens were considered as the presence of mucin pools and lacking neoplastic epithelium in specimens was defined as acellular mucin [20].

2.5. Evaluation and Follow-Up. During neoadjuvant CRT, acute toxicities were evaluated at each visit according to the Common Terminology Criteria for Adverse Events (CTCAE), version 4.03. In this study, anemia was defined as hemoglobin $(\mathrm{Hb})$ level $<10 \mathrm{~g} / \mathrm{dL}$. We evaluated post-CRT response approximately 6-10 weeks after completion of CRT. Digital rectal examinations, colonoscopy, CEA test, abdominal and chest $\mathrm{CT}$, and pelvic MRI were used for postCRT clinical assessment. MRI was used for evaluation of post-CRT locoregional stage, and CT was used for assessment of distant metastasis. Cancer restaging was recorded according to the 7th edition American Joint Committee on Cancer staging system. Post-CRT ycT0 stage was defined as no tumor in the rectal wall; ycT1 stage was defined as a tumor confined to the submucosa; ycT2 stage was defined as 
a tumor that had invaded the muscularis propria; and $\mathrm{ycN}$ stage 0 was defined as the absence of metastatic lymph nodes. Using T2-weighted MR images, a low to intermediate signalintensity lesion was considered as a residual tumor; a metastatic lymph node was considered as a size threshold of $>5 \mathrm{~mm}$ in short axis with heterogeneous signal or ill-defined margins.

After surgery, patients visited the outpatient department every 3 months in the initial 2 years and then once every 6 months to date. We defined tumor recurrence within the pelvis as a local failure and outside the pelvis as a distant failure.

2.6. Statistics. Fisher's exact test was used to compare categorical data between patients with and without a pCR. Multivariate logistic regressions were used to analyze variables with a $P$ value $<0.2$ in univariate analysis to hope for adjustments of potential colinearity. A prediction nomogram was built to show the predicted probabilities of a $\mathrm{pCR}$ rate using variables with a $P$ value $<0.05$ in multivariate analysis. A score of each independent predictive factor can be read out, and the sum of scores was converted to a probability of $\mathrm{pCR}$. A bootstrap validation method was used for internal validation of a prediction nomogram. A total of 118 bootstrap samples were randomly selected, and the concordance index (C-index) was generated to estimate the bias-corrected or overfitting-corrected predictive discriminative ability of the model.

DFS was measured from the date of starting neoadjuvant CRT to the date of any type of recurrence or last follow-up. Overall survival (OS) was defined as the time from the start of neoadjuvant CRT to death from any cause or to last follow-up. Kaplan-Meier methods were used for DFS and OS, and the log-rank test was used to compare time-to-event distributions. Data analyses were performed using the SAS version 9.3 (SAS Institute, Cary, NC) and Stata version 14. A $P$ value $<0.05$ was considered significant.

\section{Results}

The median age of the 236 patients was 63 years (range, 34-93 years). The majority of the patients had a clinical T3 primary tumor $(77.9 \%)$ and clinical nodal metastasis $(84.7 \%)$. Most patients received a total radiation dose of $50 \mathrm{~Gy}$ in 25 fractions (75.8\%). Forty-five patients were treated with three-dimensional conformal radiation therapy, and 191 patients were treated with intensity-modulated radiation therapy. The median number of cycles of chemotherapy was seven (range, 5-9), with 80 patients (33.9\%) receiving at least seven cycles of chemotherapy. The median number of post-CRT CEA was $2.2 \mathrm{ng} / \mathrm{mL}$, ranging from 0.48 to $197.5 \mathrm{ng} / \mathrm{mL}$. Therefore, $2 \mathrm{ng} / \mathrm{mL}$ was selected as the cutoff value for post-CRT CEA level. Table 1 summarizes the characteristics of patients with and without a pCR. In the retrospective cohort, 56 patients $(23.7 \%)$ achieved a pCR. Among the pCR patients, 12 patients (21.4\%) were found to have acellular mucin pools. There was no local or distant recurrence in $\mathrm{pCR}$ patients with acellular mucin and two
TABLE 1: Clinical characteristics of patients with locally advanced rectal cancer after trimodality treatment.

\begin{tabular}{|c|c|c|c|}
\hline & pCR (\%) & $\begin{array}{l}\text { Non-pCR } \\
(\%)\end{array}$ & $\begin{array}{c}P \\
\text { value* }\end{array}$ \\
\hline All patients, no. & $56(23.7)$ & $180(76.3)$ & - \\
\hline Gender & & & 0.4139 \\
\hline Male & $34(22.1)$ & $120(77.9)$ & \\
\hline Female & $22(26.8)$ & $60(73.2)$ & \\
\hline Age at diagnosis & & & 0.3590 \\
\hline$\leq 60$ & $21(20.8)$ & $80(79.2)$ & \\
\hline$>60$ & $35(25.9)$ & $100(74.1)$ & \\
\hline Location of tumor & & & 0.1564 \\
\hline Upper & $18(19.0)$ & $77(81.0)$ & \\
\hline Middle/low & $38(27.0)$ & $103(73.0)$ & \\
\hline Grade & & & 0.0577 \\
\hline Well differentiated & $0(0)$ & $16(100)$ & \\
\hline Moderate differentiated & $53(25.0)$ & $159(75.0)$ & \\
\hline Poor differentiated & $3(37.5)$ & $5(62.5)$ & \\
\hline Clinical $\mathrm{T}$ stage & & & 0.1964 \\
\hline $\mathrm{T} 2$ & $4(30.8)$ & $9(69.2)$ & \\
\hline T3 & $47(25.5)$ & $137(74.5)$ & \\
\hline $\mathrm{T} 4$ & $5(12.8)$ & $34(87.2)$ & \\
\hline Clinical N stage & & & 0.0202 \\
\hline No & $14(39.0)$ & $22(61.0)$ & \\
\hline $\mathrm{N}+$ & $42(21.0)$ & $158(79.0)$ & \\
\hline Radiation dose (cGy) & & & 0.0772 \\
\hline$\leq 4500$ & $5(11.4)$ & $39(88.6)$ & \\
\hline$>5000$ & $51(88.6)$ & $141(11.4)$ & \\
\hline \multicolumn{4}{|l|}{ Chemotherapy } \\
\hline FOLFOX & $40(28.4)$ & $101(71.6)$ & 0.0412 \\
\hline Fluoropyridine & $16(16.8)$ & $79(83.2)$ & \\
\hline $\begin{array}{l}\text { Cycles of pre-OP } \\
\text { chemotherapy }\end{array}$ & & & 0.5215 \\
\hline$<7$ & $39(25)$ & $117(75)$ & \\
\hline$\geq 7$ & $17(21.3)$ & $63(78.7)$ & \\
\hline Pre-CRT CEA (ng/mL) & & & 0.0674 \\
\hline$\leq 5$ & $40(27.8)$ & $104(72.2)$ & \\
\hline$>5$ & $16(17.4)$ & $76(82.6)$ & \\
\hline Anemia during CRT & & & 0.0159 \\
\hline $\mathrm{Hb}(\mathrm{g} / \mathrm{dL})>10$ & $47(28.0)$ & $121(72.0)$ & \\
\hline $\mathrm{Hb}(\mathrm{g} / \mathrm{dL}) \leq 10$ & $9(13.2)$ & $59(86.8)$ & \\
\hline Leukopenia during CRT & & & 0.1113 \\
\hline $\mathrm{WBC}>3000(/ \mu \mathrm{L})$ & $29(20.3)$ & $114(79.7)$ & \\
\hline $\mathrm{WBC} \leq 3000(/ \mu \mathrm{L})$ & $27(29.4)$ & $65(70.6)$ & \\
\hline RT to surgery interval & & & 0.0081 \\
\hline$\leq 8$ weeks & $11(13.6)$ & $70(86.4)$ & \\
\hline$>8$ weeks & $45(29.0)$ & $110(71.0)$ & \\
\hline ycT stage & & & 0.0734 \\
\hline T0 & $4(33.3)$ & $8(66.7)$ & \\
\hline $\mathrm{T} 1$ & $1(11.1)$ & $9(88.9)$ & \\
\hline $\mathrm{T} 2$ & $8(32)$ & $17(68)$ & \\
\hline $\mathrm{T} 3$ & $35(26.1)$ & $99(73.9)$ & \\
\hline $\mathrm{T} 4$ & $8(14.3)$ & $48(85.7)$ & \\
\hline $\mathrm{ycN}$ stage & & & 0.1515 \\
\hline No & $30(20.5)$ & $116(79.5)$ & \\
\hline $\mathrm{N}+$ & $26(28.9)$ & $64(71.1)$ & \\
\hline Post-CRT CEA (ng/mL) & & & 0.0285 \\
\hline$\leq 2$ & $9(45)$ & $11(55)$ & \\
\hline$>2$ & $47(21.8)$ & $169(78.2)$ & \\
\hline
\end{tabular}

CEA, carcinoembryonic antigen; CRT, chemoradiation therapy; FOLFOX, fluorouracil, leucovorin, and oxaliplatin; $\mathrm{Hb}$, hemoglobin; $\mathrm{OP}$, operative; pCR, pathological complete response; RT, radiation therapy; WBC, white blood cell; ycT stage: clinical tumor stage after chemoradiotherapy; ycN stage: clinical nodal stage after chemoradiotherapy. ${ }^{*}$ Fisher's exact test. 
pCR patients without mucin developed tumor recurrence. Univariate analysis revealed that a negative nodal stage $(P=0.0202)$, CRT with FOLFOX-based chemotherapy $(P=0.0412)$, high $\mathrm{Hb}$ levels $(>10 \mathrm{~g} / \mathrm{dL})$ during neoadjuvant CRT $(P=0.0159)$, long interval $(>8$ weeks) between radiotherapy and surgery $(P=0.0081)$, and post-CRT CEA levels $\leq 2 \mathrm{ng} / \mathrm{mL}(P=0.0285)$ were favorable predictors of a pCR.

The results of multivariate logistic regression are shown in Table 2. The analysis revealed that patients with a negative nodal stage (odds ratio $(\mathrm{OR})=3.2,95 \%$ confidence interval $(\mathrm{CI})=1.279-8.410, P=0.0135)$, with high $\mathrm{Hb}$ levels $(>10 \mathrm{~g} /$ $\mathrm{dL})$ during neoadjuvant $\mathrm{CRT} \quad(\mathrm{OR}=3.067, \quad 95 \%$ $\mathrm{CI}=1.251-8.187, \quad P=0.0125)$, receiving FOLFOX-based neoadjuvant CRT $(\mathrm{OR}=5.385,95 \% \mathrm{CI}=1.699-17.688$, $P=0.0044)$, with a long interval ( $>8$ weeks) between radiotherapy and surgery $(\mathrm{OR}=1.135,95 \% \mathrm{CI}=1.021-5.712$, $P=0.0469)$, and with post-CRT CEA levels $\leq 2 \mathrm{ng} / \mathrm{mL}$ $(\mathrm{OR}=2.891,95 \% \mathrm{CI}=1.156-7.369, P=0.0233)$ were more likely to achieve a pCR. In addition, we compared a combination of FOLFOX-based neoadjuvant CRT and a longer interval of $>8$ weeks with a combination of fluoropyrimidine-based neoadjuvant CRT and a shorter interval of $\leq 8$ weeks; FOLFOX regimen plus a longer interval was revealed to be an independent predictor of a pCR (OR, 5.518, 95\% $\mathrm{CI}=1.826-18.261, P=0.0024)$. Therefore, the prediction nomogram (Figure 1) was constructed to predict pCR by incorporating the four significant predictors: (I) FOLFOXbased neoadjuvant CRT with a longer interval of $>8$ weeks; (II) $\mathrm{Hb}$ level during neoadjuvant CRT; (III) post-CRT CEA level; and (IV) clinical N stage. Each predictor was assigned a score on the point scale. We could draw a vertical line downwards from the total point to obtain the probability of pCR rates. The C-index was 0.74 (95\% confidence interval $0.65-0.84$ ), which was used to test the discriminative ability of the model. The goodness-of-fit of the model was evaluated by the calibration curve (Figure 2), which revealed that the predicted probabilities of $\mathrm{pCR}$ fit well to the observed probabilities of pCR.

Figure 3 shows the percentage of patients with a pCR after different intervals between radiotherapy and surgery. Overall, the pCR rate increased as the radiotherapy-surgery interval was prolonged from $11.5 \%$ (5-6 week interval) to $36.6 \%$ ( $>13$ week interval). The pCR rate reached a plateau after an interval of $>13$ weeks.

3.1. Patient Outcomes. The median follow-up time was 36 months (range, 6-90 months). In this cohort, 35 of 236 patients (14.8\%) died. The estimated 3- and 5-year OS rates for all patients were $85.4 \%$ and $78.1 \%$, respectively. Patients who achieved a pCR had greater survival than those who did not (Figure 4(a); $P=0.0045)$. The 3- and 5-year OS rates were both $97.9 \%$ for patients with a pCR, whereas they were $81.7 \%$ and $72.9 \%$, respectively, for patients without a pCR. The 3- and 5-year DFS rates for all patients were $79.3 \%$ and $75.5 \%$, respectively. Patients with a pCR had higher DFS than those without a pCR (Figure 4(b); $P=0.0015$ ). The 3 and 5-year DFS were both $95.5 \%$ for the pCR group, whereas they were $74.3 \%$ and $69.7 \%$, respectively, for the non-pCR group.

3.2. Failure Patterns. Table 3 shows that 2 patients $(3.6 \%)$ and 42 patients $(23.3 \%$ ) from the pCR and non-pCR groups, respectively, developed tumor recurrence. In the pCR group, one patient developed local recurrence and bilateral adrenal metastases at 7 months after neoadjuvant CRT completion. Although FOLFIRI plus bevacizumab was administrated, she died of cancer progression at 3 months after the introduction of FOLFIRI plus bevacizumab. The other pCR patient had liver metastases at 15 months after neoadjuvant CRT. He received FOLFIRI plus bevacizumab, and tumor remained under control for over 8 months after liver metastases. In the non-pCR group, the risk of distant metastasis $(13.2 \%)$ was higher than that of locoregional recurrence (6.7\%). The most common sites for distant metastases in the non-pCR group were the liver $(n=15)$, lung $(n=12)$, and peritoneum $(n=4)$.

\section{Discussion}

For patients with LARC following neoadjuvant CRT, patients achieving a pCR have had higher survival rates than those who did not $[5,21]$. In this study, patients who achieved a pCR had a better OS and DFS compared with those who did not. We found that patients with a clinically negative nodal disease, without anemia during treatment, with a long interval ( $>8$ weeks) between radiotherapy and surgery, with post-CRT CEA levels $\leq 2 \mathrm{ng} / \mathrm{mL}$, and receiving a FOLFOX-based regimen were more likely to achieve a pCR to CRT. Furthermore, patients who received a FOLFOXbased regimen plus a long interval ( $>8$ weeks) between radiotherapy and surgery achieved a high pCR rate, which was consistent with that of our previous study [5]. We previously reported that extending FOLFOX and prolonging the radiotherapy-surgery interval resulted in a high $\mathrm{pCR}$ rate (31.6\%) for patients with LARC undergoing neoadjuvant CRT.

We reported a higher $\mathrm{pCR}$ rate compared with that reported by the German rectal trial (22.5\% vs. $8 \%)$ [1]. This improvement in the $\mathrm{pCR}$ rate might be because of biweekly FOLFOX administration during and after the whole course of irradiation in our cohort compared with bolus 5-FU only during radiotherapy in the German rectal trial. In this study, patients receiving FOLFOX-based neoadjuvant CRT had a significantly higher pCR rate than those undergoing fluoropyrimidine-based neoadjuvant CRT (27.2\% vs. $16.7 \%$; $P=0.0411)$. This is supported by the results of the German CAO/ARO/AIO-04 trial, which showed pCR rates of $17 \%$ and $13 \%(\mathrm{OR}, 1.40 ; 95 \% \mathrm{CI}, 1.02-1.92 ; P=0.038)$ in the 5 FU plus oxaliplatin group and fluorouracil group, respectively [22]. Another reason for a higher pCR rate in our cohort might be that most patients $(88.9 \%)$ in the fluoropyrimidine-based neoadjuvant CRT group received continuous capecitabine. Patients receiving 5-FU or capecitabine continuously during radiotherapy have shown improved pCR rates compared with those receiving bolus 5- 
TABLE 2: Independent clinical parameters significantly associated with a pCR.

\begin{tabular}{|c|c|c|c|}
\hline Parameter & OR & $95 \% \mathrm{CI}$ & $P$ value* \\
\hline Chemotherapy (FOLFOX vs. non-FOLFOX) & 5.385 & $1.699-17.688$ & 0.0044 \\
\hline Clinical N stage (N0 vs. N1/2) & 3.200 & $1.279-8.410$ & 0.0135 \\
\hline Anemia during CRT $(\mathrm{Hb}>10$ vs. $\mathrm{Hb} \leq 10)$ & 3.067 & $1.251-8.187$ & 0.0125 \\
\hline Post-CRT CEA ( $\leq 2$ vs. $>2$ ) & 2.891 & $1.156-7.369$ & 0.0233 \\
\hline RT to surgery interval ( $>8$ weeks vs. $\leq 8$ weeks) & 1.135 & $1.021-5.712$ & 0.0469 \\
\hline Gender (male vs. female) & 0.607 & $0.291-1.265$ & 0.1823 \\
\hline Age ( $\leq 60$ vs. $>60)$ & 0.680 & $0.329-1.265$ & 0.2850 \\
\hline Location of tumor (middle/low vs. upper) & 1.895 & $0.911-4.068$ & 0.0877 \\
\hline Grade (WD/MD vs. PD) & 0.349 & $0.064-2.113$ & 0.2373 \\
\hline Clinical T stage (T2/3 vs. T4) & 1.810 & $0.615-6.186$ & 0.2907 \\
\hline Radiation dose ( $<5000$ vs. $\geq 5000)$ & 0.626 & $0.186-1.799$ & 0.3972 \\
\hline Pre-CRT CEA ( $\leq 5$ vs. $>5$ ) & 1.761 & $0.861-3.727$ & 0.1221 \\
\hline Leukopenia during $\mathrm{CRT}(\mathrm{WBC}>3000$ vs. $\mathrm{WBC} \leq 3000)$ & 0.833 & $0.378-1.826$ & 0.6479 \\
\hline Cycles of pre-OP chemotherapy $(\geq 7$ vs. $<7)$ & 1.037 & $0.656-1.625$ & 0.8732 \\
\hline yc'T stage (T0-2 vs. T3-4) & 1.872 & $0.886-4.110$ & 0.1008 \\
\hline $\mathrm{ycN}$ stage (N0 vs. N1/2) & 1.624 & $0.799-3.311$ & 0.1788 \\
\hline
\end{tabular}

CEA, carcinoembryonic antigen; CRT, chemoradiation therapy; FOLFOX, fluorouracil, leucovorin, and oxaliplatin; Hb, hemoglobin; OP, operative; pCR, pathological complete response; RT, radiation therapy; WBC, white blood cell; ycT stage: clinical tumor stage after chemoradiotherapy; ycN stage: clinical nodal stage after chemoradiotherapy. ${ }^{*}$ Logistic regression.

Chemotherapy
Non-FOLFOX FOLFOX

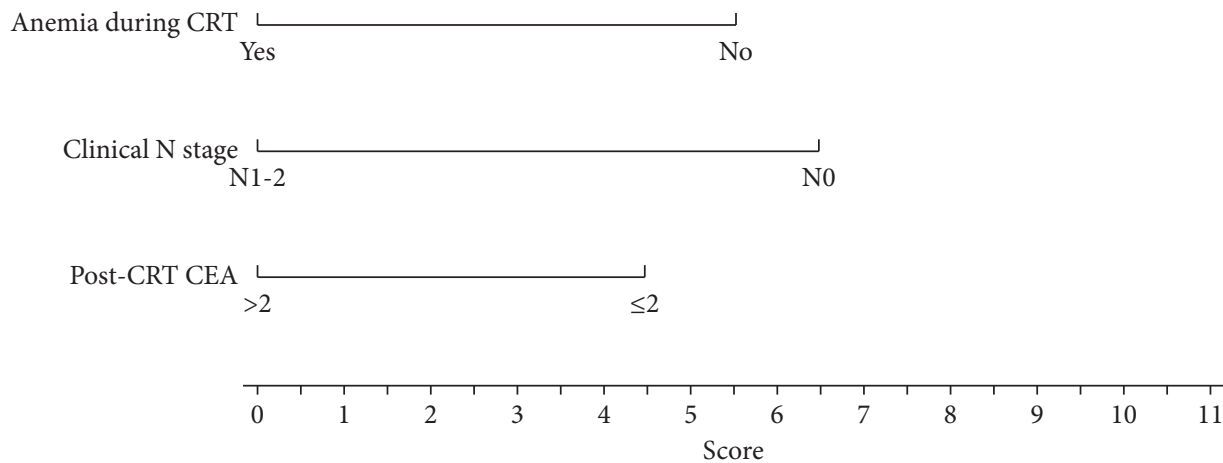

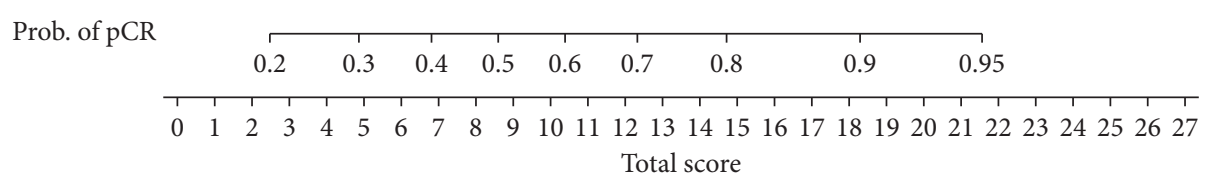

FIGURE 1: Nomogram developed for prediction of pathological complete response (pCR) rates. A score of each predictive factor can be read out at the top scale, and the sum of scores is converted to a probability of pCR. CEA, carcinoembryonic antigen; CRT, chemoradiation therapy; pCR, pathological complete response.

FU $[4,15]$. Therefore, our overall pCR rate was higher than that reported in the German rectal trial.

Clinical $\mathrm{T}$ stages, radiation doses, and pre-CRT serum CEA levels were not predictive factors of a pCR in the current study, although some studies have shown that pretreatment CEA level was a crucial pCR predictor $[12,14]$. Furthermore, a smaller tumor size and a higher radiation dose have been reported to be associated with increased pCR rates $[12,13,16]$.

In colorectal cancer (CRC), positive pathological nodes have been associated with poor prognosis [13, 21, 23]. For patients with LARC following neoadjuvant CRT, pretreatment negative lymph nodes have been correlated with increased pCR rates $[13,21]$. Clinical $N$ stage is not always correlated with pathological $\mathrm{N}$ stage [24]. It might not be easy to differentiate reactive nodes from metastatic nodes in current image studies. However, positive pretreatment nodal status usually represents tumor progression and aggression. In our cohort, $39.4 \%$ of the patients with clinically negative $\mathrm{N}$ stage achieved a pCR, whereas only $19.3 \%$ of the patients with pretreatment positive $\mathrm{N}$ stage achieved a pCR. Therefore, clinical non- $\mathrm{N}$ stage was considered to be a potential indicator of pCR in our study.

$\mathrm{Hb}$ levels have been associated with oncologic outcomes in numerous malignancies including head and neck cancer, cervical cancer, and CRC [25-27]. In general, anemia is 


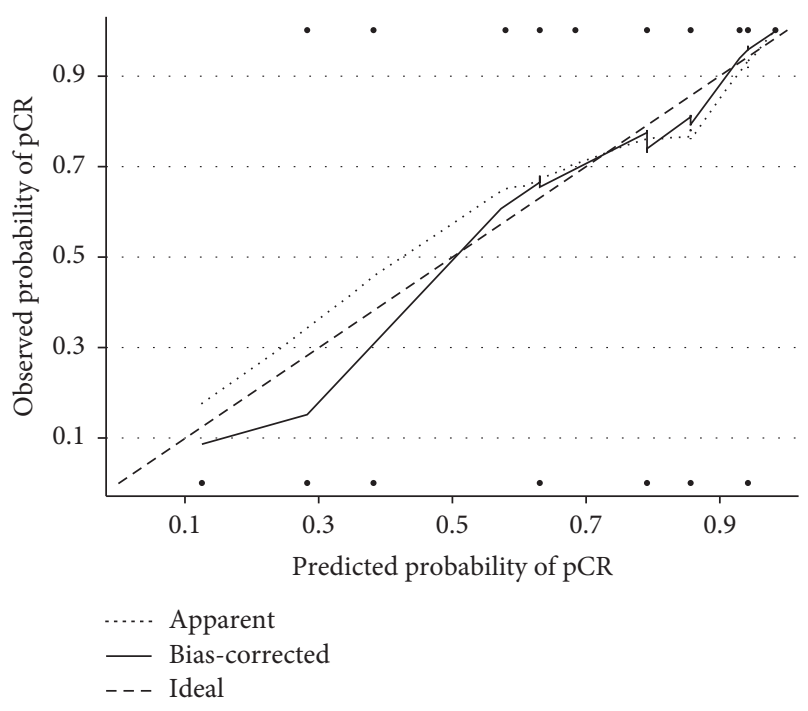

FIGURE 2: Calibration curve of observed and predicted probabilities. The $x$ axis is the predicted probabilities measured by the final logistic regression model and the $y$ axis is the actual probabilities. The long-dashed line represents an ideal nomogram whose predicted outcome perfectly corresponds to the actual outcome. The solid line (bias-corrected) represents the bootstrap-corrected performance of our nomogram, and the short-dashed line represents apparent accuracy of the nomogram. The apparent and biascorrected line fell approximately along the ideal line, which indicates that the calculated by the nomogram accurately represents the actual prediction of pathological complete response (pCR) for rectal cancer in both the primary and validation cohorts.

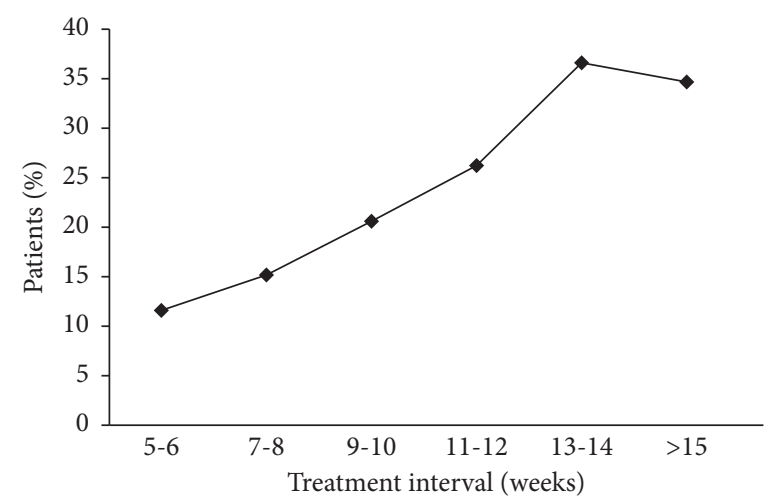

\begin{tabular}{lcccccc}
$\qquad$ & $\bullet$ & pCR rate & & & & \\
\hline Weeks & $5-6$ & $7-8$ & $9-10$ & $11-12$ & $13-14$ & $>15$ \\
\hline pCR $(\mathrm{N})$ & 3 & 8 & 8 & 16 & 11 & 9 \\
Non-pCR $(\mathrm{N})$ & 23 & 45 & 31 & 45 & 19 & 17 \\
pCR rate (\%) & 11.5 & 15.1 & 20.5 & 26.2 & 36.6 & 34.6 \\
\hline
\end{tabular}

FIgURE 3: Percentage of patients with a pathological complete response (pCR) at different intervals between radiotherapy and surgery.

defined as $\mathrm{Hb}$ level of $\leq 12 \mathrm{~g} / \mathrm{dL}$ in women and $\leq 13.5 \mathrm{~g} / \mathrm{dL}$ in men according to the Third National Health and Nutrition Examination Survey [28]. However, we defined anemia as $\mathrm{Hb}$ level of $\leq 10 \mathrm{~g} / \mathrm{dL}$ according to the CTCAE 4.03 because we attempted to correlate treatment toxicity with pCR achievement. The role of pretreatment anemia in treatment response and tumor control has been reported [27, 29]. Berardi et al. identified that $\mathrm{Hb}$ level of $>12 \mathrm{~g} / \mathrm{dL}$ was associated with higher DFS and tumor downstaging for patients with rectal cancer receiving neoadjuvant radiotherapy with or without chemotherapy in a retrospective review of 317 patients [29]. Khan et al. studied clinical parameters of 463 patients with LARC and found that a pretreatment $\mathrm{Hb}$ level of $>12 \mathrm{~g} / \mathrm{dL}$ was associated with response to neoadjuvant CRT and risk of local recurrence [30]. Our study found that $\mathrm{Hb}$ level of $>10 \mathrm{~g} / \mathrm{dL}$ during neoadjuvant CRT was a significant predictor of increased pCR rates. To our knowledge, this is the first study to correlate treatmentrelated anemia with probabilities of $\mathrm{PCR}$ to neoadjuvant CRT in LARC.

In general, severe toxicity might reflect favorable treatment response except for achievement rates. However, patients with low $\mathrm{Hb}$ levels during neoadjuvant CRT experienced unfavorable treatment response in our study. Because anemia has been correlated to tumor hypoxia, angiogenesis, and resistance to chemotherapy and radiotherapy [7, 31], we suggested that high $\mathrm{Hb}$ levels during treatment could sensitize tumor cells to radiation due to improved oxygenation. This might explain why severe hematologic toxicity did not translate into better treatment response.

We found patients receiving FOLFOX-based neoadjuvant CRT to be more likely to achieve a pCR compared with those receiving fluoropyrimidine-based neoadjuvant CRT $(27.2 \%$ vs. $16.7 \%$; $P=0.0411)$. Our results are supported by 2 phase III randomized studies. The phase III German CAO/ARO/AIO-04 trial demonstrated that addition of oxaliplatin in a neoadjuvant therapy improved the pCR rate and DFS compared with fluorouracil-based neoadjuvant CRT $[22,32]$. Another phase III Chinese FOWARC trial studied the combination of fluorouracil-based neoadjuvant CRT with and without oxaliplatin for patients with LARC and demonstrated that FOLFOX-based neoadjuvant CRT resulted in a higher pCR rate than 5-FU-based neoadjuvant CRT [7]. However, the effectiveness of oxaliplatin addition to fluoropyrimidine-based neoadjuvant CRT remains undetermined because some phase III trials have shown similar pCR rates between oxaliplatin-containing and fluoropyrimidine-based neoadjuvant CRT [6, 31]. This discrepancy among those trials might be because of various dose accumulation of oxaliplatin in each trial, the different treatment duration of delivering oxaliplatin during irradiation or after completion of irradiation, and possibly different treatment response between patients of different ethnicities.

The interval between radiotherapy and surgery has been associated with a pCR to neoadjuvant CRT [33, 34]. Intentional prolongation of the interval between radiotherapy and surgery has been investigated in 2 randomized studies. The Lyon R90-01 trial compared outcomes for intervals of 2 and 6 to 8 weeks between radiotherapy and surgery and demonstrated that a longer interval resulted in favorable pathological downstaging [35]. The Istanbul R-01 trial investigated the outcomes of surgery at 4 and 8 weeks after 


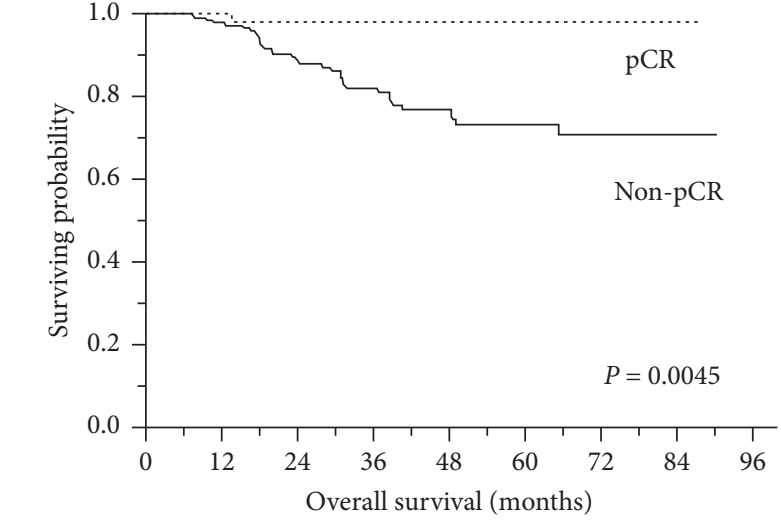

$\begin{array}{rcccccccc}\text { No. at risk } 56 & 51 & 38 & 29 & 16 & 7 & 5 & 2 & \text { pCR } \\ 180 & 161 & 114 & 84 & 63 & 33 & 18 & 8 & \text { Non-pCR }\end{array}$

(a)

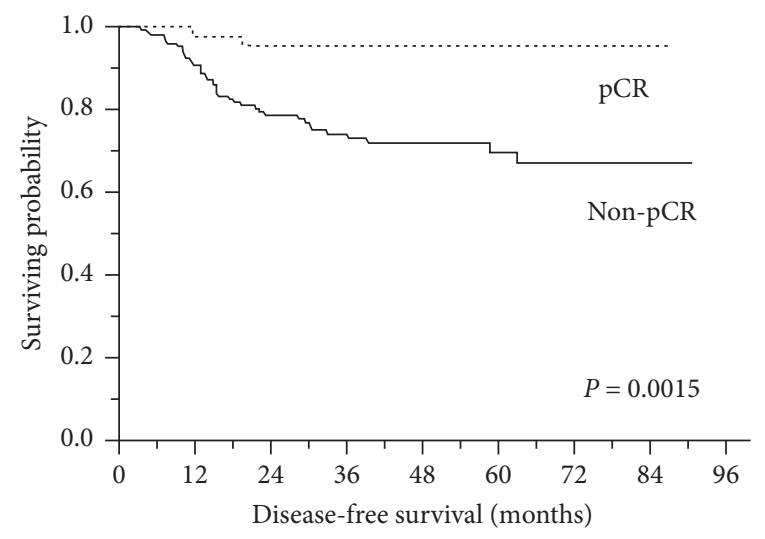

$\begin{array}{lllllllll}\text { No. at risk } 56 & 51 & 37 & 29 & 16 & 7 & 5 & 2 & \text { pCR }\end{array}$ $\begin{array}{lllllllll}180 & 146 & 95 & 69 & 54 & 30 & 15 & 7 & \text { Non-pCR }\end{array}$

(b)

FIGURE 4: Overall and disease-free survival in patients with locally advanced rectal cancer. Overall survival (a) and disease-free survival (b) between patients with and without a pathological complete response (pCR).

TABLE 3: Failure patterns among patients with locally advanced rectal cancer after trimodality treatment.

\begin{tabular}{lcc}
\hline Recurrence & pCR & Non-pCR \\
\hline Local/regional only & 0 & $12 / 180(6.7 \%)$ \\
Distant only & $1 / 56(1.8)$ & $24 / 180(13.2 \%)$ \\
Local/regional/distant & $1 / 56(1.8)$ & $6 / 180(3.3 \%)$ \\
Total & $2 / 56(3.6)$ & $42 / 180(23.3 \%)$ \\
\hline
\end{tabular}

pCR, pathological complete response.

neoadjuvant CRT and found no difference in tumor regression between the 2 intervals [36]. However, we observed a pCR rate of $>33 \%$ at an interval of 13 weeks. A longer interval ( $>8$ weeks) seemed to be associated with a higher chance of pCR. This is supported by several retrospective studies [5, 33, 37]. Sloothaak et al. reviewed 1593 patients with LARC and found that delaying surgery until the 10th to 11th week after the end of radiotherapy achieved the highest pCR rate [34]. Rombouts et al. studied 1073 patients with LARC and revealed that radiotherapy-surgery intervals of 9 to 12 weeks resulted in a high pCR rate [37].

Some studies have reported that post-CRT clinical factors were associated with pCR $[23,38]$. In the current study, several post-CRT variables were analyzed and post-CRT CEA $\leq 2 \mathrm{ng} / \mathrm{mL}$ was an independent predictor of increased pCR rates. CEA is associated with prognosis and treatment response in patients with LARC treated with neoadjuvant CRT [39]. Deceased post-CRT CEA levels have been reported to be associated with increased pCR rates in LARC patients following neoadjuvant CRT $[38,40]$. Lowering postCRT CEA levels might imply decreased tumor burdens and therefore, a favorable response to CRT was achieved.

We established the prediction nomogram model to predict a pCR for LARC patients following neoadjuvant CRT. FOLFOX-based neoadjuvant CRT with a longer interval of $>8$ weeks, $\mathrm{Hb}>10 \mathrm{~g} / \mathrm{dL}$ during neoadjuvant CRT, a clinical N0 stage, and post-CRT CEA $\leq 2 \mathrm{ng} / \mathrm{mL}$ were significant predictors of increased $\mathrm{pCR}$ rates. This data could facilitate patient selection and help clinicians to identify the right patients who may benefit most from watch-and-wait strategies. However, validating the prediction nomogram on an independent cohort would be mandatory. Although we did not have an independent cohort for external validation of the nomogram, we carried out a bootstrap method for internal validation of our model. The $\mathrm{C}$-index of our model was 0.74 , which indicated that our nomogram had a high predictive accuracy of $74 \%$. Thus, our model wound be helpful in clinical use.

This study had several limitations. First, this was a retrospective study, and heterogeneity in patient selection and treatment decision by individual physicians may have influenced the results. Second, we only reported a correlation between $\mathrm{Hb}$ levels and a pCR, but other associated inflammation-based parameters, such as serum albumin, C-reactive protein, and neutrophil and lymphocyte counts, were not completely collected. These inflammation-related factors have been reported to be associated with tumor response to neoadjuvant CRT for LARC [41, 42]. Third, MRI features were not included for analysis due to some missing data, though evidence has shown MRI features to be predictive factors of $\mathrm{pCR}$ in LARC patients $[43,44]$.

\section{Conclusions}

Our results demonstrated that clinical negative nodal diseases, $\mathrm{Hb}$ levels $>10 \mathrm{~g} / \mathrm{dL}$ during neoadjuvant CRT, FOLFOX-based neoadjuvant CRT with a longer radiotherapy-surgery interval, and post-CRT CEA levels $\leq 2 \mathrm{ng} / \mathrm{mL}$ resulted in a greater likelihood of a $\mathrm{pCR}$. The nomogram model to predict a pCR rate for LARC patients following neoadjuvant CRT would be potential in clinical implication; however, further prospective, randomized large-scale studies are warranted to validate our results. 


\section{Data Availability}

The data used to support the findings of this study are included within the article, and the data sources are available from the corresponding author upon request.

\section{Conflicts of Interest}

The authors declare that they have no conflicts of interest.

\section{Acknowledgments}

This work was supported by grants through funding from the Ministry of Science and Technology (MOST108-2321-B037-001, MOST107-2321-B-037-003, MOST107-2314-B037-116, MOST107-2314-B-037-022-MY2, and MOST1072314-B-037-023-MY2), the Ministry of Health and Welfare (MOHW107-TDU-B-212-123006, MOHW107-TDU-B212-114026B, MOHW108-TDU-B-212-133006, and MOHW108-TDU-B-212-124026) funded by Health and Welfare Surcharge of Tobacco Products, the Kaohsiung Medical University Hospital (KMUH105-5M41, KMUH107-7R28, KMUH107-7R29, KMUH107-7R30, KMUH107-7M22, KMUH107-7M23, KMUHS10701, KMUHS10801, KMUHS10804, and KMUHS10807), and Center for Cancer Research, Kaohsiung Medical University (KMU-TC108A04). In addition, this study was supported by the Grant of Taiwan Precision Medicine Initiative and Biomarker Discovery in Major Diseases of Taiwan Project (AS-BD-108-1), Academia Sinica, Taiwan, ROC.

\section{References}

[1] R. Sauer, H. Becker, W. Hohenberger et al., "Preoperative versus postoperative chemoradiotherapy for rectal cancer," New England Journal of Medicine, vol. 351, no. 17, pp. 17311740, 2004.

[2] D. Sebag-Montefiore, R. J. Stephens, R. Steele et al., "Preoperative radiotherapy versus selective postoperative chemoradiotherapy in patients with rectal cancer (MRC CR07 and NCIC-CTG C016): a multicentre, randomised trial," The Lancet, vol. 373, no. 9666, pp. 811-820, 2009.

[3] M. S. Roh, L. H. Colangelo, M. J. O'Connell et al., "Preoperative multimodality therapy improves disease-free survival in patients with carcinoma of the rectum: NSABP R-03," Journal of Clinical Oncology, vol. 27, no. 31, pp. 5124-5130, 2009.

[4] A. Hartley, K. F. Ho, C. McConkey, and J. I. Geh, "Pathological complete response following pre-operative chemoradiotherapy in rectal cancer: analysis of phase II/III trials," The British Journal of Radiology, vol. 78, no. 934, pp. 934-938, 2005.

[5] C.-M. Huang, M.-Y. Huang, H.-L. Tsai et al., "An observational study of extending FOLFOX chemotherapy, lengthening the interval between radiotherapy and surgery, and enhancing pathological complete response rates in rectal cancer patients following preoperative chemoradiotherapy," Therapeutic Advances in Gastroenterology, vol. 9, no. 5, pp. 702-712, 2016.

[6] J.-P. Gérard, D. Azria, S. Gourgou-Bourgade et al., "Clinical outcome of the ACCORD 12/0405 PRODIGE 2 randomized trial in rectal cancer," Journal of Clinical Oncology, vol. 30, no. 36, pp. 4558-4565, 2012.

[7] Y. Deng, P. Chi, P. Lan et al., "Modified FOLFOX6 with or without radiation versus fluorouracil and leucovorin with radiation in neoadjuvant treatment of locally advanced rectal cancer: initial results of the Chinese FOWARC multicenter, open-label, randomized three-arm phase III trial," Journal of Clinical Oncology, vol. 34, no. 27, pp. 3300-3307, 2016.

[8] C. M. Huang, M. Y. Huang, C. J. Ma et al., "Neoadjuvant FOLFOX chemotherapy combined with radiotherapy followed by radical resection in patients with locally advanced colon cancer," Radiation Oncology, vol. 12, no. 1, p. 48, 2017.

[9] M.-Y. Huang, J.-J. Huang, C.-M. Huang et al., "Relationship between expression of proteins ERCC1, ERCC2, and XRCC1 and clinical outcomes in patients with rectal cancer treated with FOLFOX-based preoperative chemoradiotherapy," World Journal of Surgery, vol. 41, no. 11, pp. 2884-2897, 2017.

[10] M. Y. Huang, C. H. Wu, C. M. Huang et al., "DPYD, TYMS, TYMP, TK1, and TK2 genetic expressions as response markers in locally advanced rectal cancer patients treated with fluoropyrimidine-based chemoradiotherapy," BioMed Research International, vol. 2013, Article ID 931028, 10 pages, 2013.

[11] N. K. Kim and H. Hur, "New perspectives on predictive biomarkers of tumor response and their clinical application in preoperative chemoradiation therapy for rectal cancer," Yonsei Medical Journal, vol. 56, no. 6, pp. 1461-1477, 2015.

[12] E. Al-Sukhni, K. Attwood, D. M. Mattson, E. Gabriel, and S. J. Nurkin, "Predictors of pathologic complete response following neoadjuvant chemoradiotherapy for rectal cancer," Annals of Surgical Oncology, vol. 23, no. 4, pp. 1177-1186, 2016.

[13] M. L. Garland, R. Vather, N. Bunkley, M. Pearse, and I. P. Bissett, "Clinical tumour size and nodal status predict pathologic complete response following neoadjuvant chemoradiotherapy for rectal cancer," International Journal of Colorectal Disease, vol. 29, no. 3, pp. 301-307, 2014.

[14] U. Wallin, D. Rothenberger, A. Lowry, R. Luepker, and A. Mellgren, "CEA-a predictor for pathologic complete response after neoadjuvant therapy for rectal cancer," Diseases of the Colon and Rectum, vol. 56, no. 7, pp. 859-868, 2013.

[15] P. Sanghera, D. W. Y. Wong, C. C. McConkey, J. I. Geh, and A. Hartley, "Chemoradiotherapy for rectal cancer: an updated analysis of factors affecting pathological response," Clinical Oncology, vol. 20, no. 2, pp. 176-183, 2008.

[16] M. Mohiuddin, W. F. Regine, W. J. John et al., "Preoperative chemoradiation in fixed distal rectal cancer: dose time factors for pathological complete response," International Journal of Radiation Oncology*Biology*Physics, vol. 46, no. 4, pp. 883$888,2000$.

[17] A. Habr-Gama, R. O. Perez, W. Nadalin et al., "Operative versus nonoperative treatment for stage 0 distal rectal cancer following chemoradiation therapy: long-term results," $A n$ nuals of Surgery, vol. 240, no. 4, pp. 711-717, 2004.

[18] J. D. Smith, J. A. Ruby, K. A. Goodman et al., "Nonoperative management of rectal cancer with complete clinical response after neoadjuvant therapy," Annals of Surgery, vol. 256, no. 6, pp. 965-972, 2012.

[19] M. Y. Huang, H. H. Lee, H. L. Tsai et al., "Comparison of efficacy and safety of preoperative Chemoradiotherapy in locally advanced upper and middle/lower rectal cancer," Radiation Oncology, vol. 13, no. 1, p. 53, 2018.

[20] J. A. Cienfuegos, J. Baixauli, F. Rotellar et al., "Clinical significance of cellular and acellular mucin pools in rectal 
carcinoma following preoperative chemoradiotherapy," Clinical and Translational Oncology, vol. 18, no. 7, pp. 714721, 2016.

[21] J. W. Huh, H. R. Kim, and Y. J. Kim, "Clinical prediction of pathological complete response after preoperative chemoradiotherapy for rectal cancer," Diseases of the Colon and Rectum, vol. 56, no. 6, pp. 698-703, 2013.

[22] C. Rödel, T. Liersch, H. Becker et al., "Preoperative chemoradiotherapy and postoperative chemotherapy with fluorouracil and oxaliplatin versus fluorouracil alone in locally advanced rectal cancer: initial results of the German CAO/ ARO/AIO-04 randomised phase 3 trial," The Lancet Oncology, vol. 13, no. 7, pp. 679-687, 2012.

[23] C. H. Park, H. C. Kim, Y. B. Cho et al., "Predicting tumor response after preoperative chemoradiation using clinical parameters in rectal cancer," World Journal of Gastroenterology, vol. 17, no. 48, pp. 5310-5316, 2011.

[24] C. Klessen, P. Rogalla, and M. Taupitz, "Local staging of rectal cancer: the current role of MRI," European Radiology, vol. 17, no. 2, pp. 379-389, 2007.

[25] C. M. Hoff, H. S. Hansen, M. Overgaard et al., "The importance of haemoglobin level and effect of transfusion in HNSCC patients treated with radiotherapy-results from the randomized DAHANCA 5 study," Radiotherapy Oncology, vol. 98, no. 1, pp. 28-33, 2011.

[26] J. Dunst, T. Kuhnt, H. G. Strauss et al., "Anemia in cervical cancers: impact on survival, patterns of relapse, and association with hypoxia and angiogenesis," International Journal of Radiation Oncology, Biology, Physics, vol. 56, no. 3, pp. 778787, 2003.

[27] H. K. van Halteren, S. Houterman, C. D. Verheij, V. E. Lemmens, and J. W. Coebergh, "Anaemia prior to operation is related with poorer long-term survival in patients with operable rectal cancer," European Journal of Surgical Oncology, vol. 30, no. 6, pp. 628-632, 2004.

[28] E. Beutler and J. Waalen, "The definition of anemia: what is the lower limit of normal of the blood hemoglobin concentration?," Blood, vol. 107, no. 5, pp. 1747-1750, 2006.

[29] R. Berardi, C. Braconi, G. Mantello et al., "Anemia may influence the outcome of patients undergoing neo-adjuvant treatment of rectal cancer," Annals of Oncology, vol. 17, no. 11, pp. 1661-1664, 2006.

[30] A. A. Khan, M. Klonizakis, A. Shabaan, and R. Glynne-Jones, "Association between pretreatment haemoglobin levels and morphometric characteristics of the tumour, response to neoadjuvant treatment and long-term outcomes in patients with locally advanced rectal cancers," Colorectal Disease, vol. 15, no. 10, pp. 1232-1237, 2013.

[31] M. J. O'Connell, L. H. Colangelo, R. W. Beart et al., "Capecitabine and oxaliplatin in the preoperative multimodality treatment of rectal cancer: surgical end points from National Surgical Adjuvant Breast and Bowel Project trial R-04," Journal of Clinical Oncology, vol. 32, no. 18, pp. 1927-1934, 2014.

[32] C. Rodel, U. Graeven, R. Fietkau et al., "Oxaliplatin added to fluorouracil-based preoperative chemoradiotherapy and postoperative chemotherapy of locally advanced rectal cancer (the German CAO/ARO/AIO-04 study): final results of the multicentre, open-label, randomised, phase 3 trial," The Lancet Oncology, vol. 16, no. 8, pp. 979-989, 2015.

[33] F. Petrelli, G. Sgroi, E. Sarti, and S. Barni, "Increasing the interval between neoadjuvant chemoradiotherapy and surgery in rectal cancer," Annals of Surgery, vol. 263, no. 3, pp. 458-464, 2016.
[34] D. A. M. Sloothaak, D. E. Geijsen, N. J. van Leersum et al., "Optimal time interval between neoadjuvant chemoradiotherapy and surgery for rectal cancer," British Journal of Surgery, vol. 100, no. 7, pp. 933-939, 2013.

[35] Y. Francois, C. J. Nemoz, J. Baulieux et al., "Influence of the interval between preoperative radiation therapy and surgery on downstaging and on the rate of sphincter-sparing surgery for rectal cancer: the Lyon R90-01 randomized trial," Journal of Clinical Oncology, vol. 17, no. 8, p. 2396, 1999.

[36] S. Saglam, D. Bugra, E. K. Saglam et al., "Fourth versus eighth week surgery after neoadjuvant radiochemotherapy in T3-4/ N0+ rectal cancer: Istanbul R-01 study," Journal of Gastrointestinal Oncology, vol. 5, no. 1, pp. 9-17, 2014.

[37] A. J. M. Rombouts, N. Hugen, M. A. G. Elferink, I. D. Nagtegaal, and J. H. W. de Wilt, "Treatment interval between neoadjuvant chemoradiotherapy and surgery in rectal cancer patients: a population-based study," Annals of Surgical Oncology, vol. 23, no. 11, pp. 3593-3601, 2016.

[38] J. Peng, J. Lin, X. Wu et al., "Clinical factors of post-chemoradiotherapy as valuable indicators for pathological complete response in locally advanced rectal cancer," Clinics, vol. 71, no. 8, pp. 449-454, 2016.

[39] J. W. Park, S.-B. Lim, D. Y. Kim et al., "Carcinoembryonic antigen as a predictor of pathologic response and a prognostic factor in locally advanced rectal cancer patients treated with preoperative chemoradiotherapy and surgery," International Journal of Radiation Oncology*Biology*Physics, vol. 74, no. 3, pp. 810-817, 2009.

[40] A. Kleiman, A. Al-Khamis, A. Farsi et al., "Normalization of CEA levels post-neoadjuvant therapy is a strong predictor of pathologic complete response in rectal cancer," Journal of Gastrointestinal Surgery, vol. 19, no. 6, pp. 1106-1112, 2015.

[41] M. Krauthamer, K. Rouvinov, S. Ariad et al., "A study of inflammation-based predictors of tumor response to neoadjuvant chemoradiotherapy for locally advanced rectal cancer," Oncology, vol. 85, no. 1, pp. 27-32, 2013.

[42] R. Carruthers, L. M. Tho, J. Brown, S. Kakumanu, E. McCartney, and A. C. McDonald, "Systemic inflammatory response is a predictor of outcome in patients undergoing preoperative chemoradiation for locally advanced rectal cancer," Colorectal Disease, vol. 14, no. 10, pp. e701-e707, 2012.

[43] K. Nie, L. Shi, Q. Chen et al., "Rectal cancer: assessment of neoadjuvant chemoradiation outcome based on radiomics of multiparametric MRI," Clinical Cancer Research, vol. 22, no. 21, pp. 5256-5264, 2016.

[44] N. Dinapoli, B. Barbaro, R. Gatta et al., "Magnetic resonance, vendor-independent, intensity histogram analysis predicting pathologic complete response after radiochemotherapy of rectal cancer," International Journal of Radiation Oncology*Biology*Physics, vol. 102, no. 4, pp. 765-774, 2018. 